International Mathematical Forum, 2, 2007, no. 28, 1363 - 1373

\title{
The Semidirect Product and the First Cohomology of Topological Groups
}

\author{
H. Sahleh \\ Department of Mathematics \\ Guilan University \\ P.O. Box 1914, Rasht, Iran \\ sahleh@guilan.ac.ir
}

\begin{abstract}
Let $Q$ be a topological group and $K$ a trivial $Q$-module. It is known that the second cohomology $H^{2}(Q, K)$ is isomorphic with the group of extensions of $Q$ by $K$. In this paper by means of semidirect product we relate the first cohomology,$H^{1}(Q, K)$, to a quotient of automorphisms of $Q$. Also we show that vanishing $H^{1}(Q, K)$ implies conjugacy of complements in the semidirect product when $K$ is abelian.
\end{abstract}

\section{Mathematics Subject Classification: 22A05}

Keywords: Cohomology of topological groups; Topological extensions; Semidirect product

\section{Introduction}

The concept of semidirect product is one of the basic notions in group theory . In recent years it has found its way into Banach algebra theory $[7,9,3]$ and categorical group [5].In this paper we use it in the category of topological groups .

Spaces are assumed to be completely regular and Hausdorff. A topological extension of $Q$ by $A$ is a short exact sequence $0 \rightarrow A \stackrel{i}{\rightarrow} G \stackrel{\pi}{\rightarrow} Q \rightarrow 0$ ,where $i$ is a topological embedding onto a closed subgroup and $\pi$ an open continuous onto homomorphism. The extension is central if $A$ is in the center of $G$. We consider extensions with a continuous section i.e. $u: Q \rightarrow G$ such that $\pi u=I d$. For example, if $Q$ is a connected locally compact group ,then any topological extension of $Q$ by a connected simply connected Lie group has a continuous section [6,theorem 2]. Notation and definitions as in [2]. By $Z Q$ 
we mean the group ring whose elements are all $\sum_{x \in Q} m_{x} x, m_{x} \in Z$.

In section 1 we describe the cohomology and semidirect product of topological groups. In section 2 we show that vanishing of $H^{1}(Q, A)$ implies that the complements in the semidirect product are conjugate.

\section{Cohomology and semidirect product of topo- logical groups}

When $Q$ is a topological group the theory of cohomology gets more interesting since we have both algebraic and topological notions of cohomology and there are different ways to combine them. In this section we are interested in the cohomology in dimensions one and two. Also we describe the semidirect product in the category of topological groups.

Let $Q$ be a topological group and $K$ an abelian topological group on which $Q$ acts continuously.

Let $C^{n}(Q, K)$ be the continuous maps $\phi: Q^{n} \rightarrow K$ with the coboundary map

$$
\left.C^{n}(Q, K) \stackrel{\delta_{n}}{\rightarrow} C^{n+1}(Q, K)\right)
$$

given by

$$
\begin{aligned}
\delta \phi\left(g_{1}, \ldots, g_{n}\right)= & g_{1} . \phi\left(g_{2}, \ldots, g_{n}\right) \\
& +\sum_{i=1}^{n-1}(-1)^{i} \phi\left(g_{1}, \ldots, g_{i} g_{i+1}, \ldots, g_{n}\right)+(-1)^{n} \phi\left(g_{1}, \ldots, g_{n-1}\right)
\end{aligned}
$$

Note that this is analogous to the inhomogeneous resolution for the discrete case[4].

Definition 1.1. The continuous group cohomology of $G$ with coefficient in $K$ is

$$
H^{n}(Q, K)=\operatorname{ker} \delta_{n} / \operatorname{Im} \delta_{n-1}
$$

Let $\operatorname{Ext}_{s}(Q, K)$ be the set of extensions of $Q$ by $A$ with a continuous section. It is known [1] ,by the Baer sum, that $\operatorname{Ext}_{s}(Q, K)$ is an abelian topological group. By [6], if $Q$ is a topological group and $K$ a trivial $Q$-module then there is an isomorphism between the second cohomology of $Q$ and the group of extensions of $Q$ by $K$ with continuous sections, namely

$$
H^{2}(Q, K) \simeq \operatorname{Ext}_{s}(Q, K)
$$

Note that if the extension $0 \rightarrow K \stackrel{i}{\rightarrow} G \stackrel{\pi}{\rightarrow} Q \rightarrow 0$ has a continuous section then $G \simeq K \times Q$, as topological spaces [1]. 


\section{Semidirect product of topological groups}

In this part we define the semidirect product in the category of topological groups. The idea is motivated by [2].

Definition 1.2 Let $K$ and $Q$ be topological groups. The semidirect product of $Q$ and $K$ is an exact sequence $0 \rightarrow K \stackrel{i}{\rightarrow} G \stackrel{\pi}{\rightarrow} Q \rightarrow 0$ with a continuous homomorphism $u: Q \rightarrow G$ such that $\pi u=I d_{Q}$. Sometimes $G$ itself is called a semidirect product of $Q$ and $A$.

Examples.

(1) A direct product $K \times Q$ is a semidirect product of $K$ by $Q$ (also $Q$ by $K)$

(2) An abelian group is a semidirect product iff it is a direct (usually called a direct sum ) since every subgroup of an abelian group is normal.

(3) cyclic groups of prime power order are not semidirect product since they can not be direct sum of two proper subgroups.

For the extensions there is a standard notion of equivalence.

Definition 1.3 Let $K$ and $Q$ be topological groups and

$$
\begin{aligned}
(e): 0 \rightarrow K \stackrel{i}{\rightarrow} G \stackrel{\pi}{\rightarrow} Q \rightarrow 0 \\
\left(e^{\prime}\right): 0 \rightarrow K \stackrel{i}{\rightarrow} G^{\prime} \stackrel{\pi}{\rightarrow} Q \rightarrow 0
\end{aligned}
$$

be two semidirect product of $Q$ and $K$ with the homomorphisms $u: Q \rightarrow G$ $, u^{\prime}: Q \rightarrow G^{\prime}$, respectively. Then $(e)$ and $\left(e^{\prime}\right)$ are equivalent, denoted by $e \sim e^{\prime}$, if there is an open continuous isomorphism $\alpha: G \rightarrow G^{\prime}$ such that the following diagram commutes

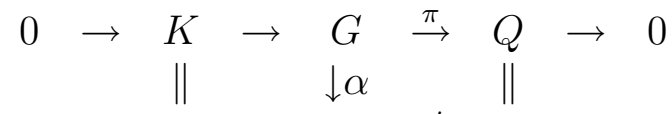

$$
\begin{aligned}
& 0 \rightarrow K \rightarrow G^{\prime} \stackrel{\pi^{\prime}}{\rightarrow} Q \rightarrow 0 \\
& \pi \alpha=\pi^{\prime}, \pi u=I d_{Q}, \pi^{\prime} u^{\prime}=I d_{Q}
\end{aligned}
$$

Remark. In definition 1.3 it is sufficient to demand that $\alpha$ be continuous isomorphism. It can be shown that $\alpha$ is open as follows: Let $U_{1}$ be a neighborhood of identity in $G$. Since $\pi$ is open and $\pi^{\prime}$ is continuous we can choose a neighborhood of identity $V_{1} \subset U_{1}$ in $G$ and a neighborhood of identity $U$ in 
$G^{\prime}$ such that $\pi^{\prime}(U) \subseteq \pi\left(V_{1}\right)$ and $\left(U \alpha\left(V_{1}\right)^{-1}\right) \cap K \subseteq U_{1}$. Then for every $u \in U$ , there is a $v \in V_{1}$ such that $\pi^{\prime}(u)=\pi(v)=\pi^{\prime} \alpha(v)$. Hence $u=q \alpha(v)$ where $q \in\left(U \alpha\left(V_{1}\right)^{-1}\right) \cap K \subseteq U_{1}$. Thus $q v \in U_{1} U_{1}$ and $\alpha(q v)=u$. We have shown that $\alpha\left(U_{1} U_{1}\right) \supseteq U$ and so is a neighborhood of identity in $G$. This is sufficient to say that $\alpha$ is open. We consider the case where $K$ is abelian.

Proposition 1.4.Let $0 \rightarrow K \stackrel{i}{\rightarrow} G \stackrel{\pi}{\rightarrow} Q \rightarrow 0$ be an extension with a continuous section $u: Q \rightarrow G$.

(1) for every $x \in G$, conjugation $\theta_{x}: K \rightarrow K$ defined by $x . a=u(x) a u(x)^{-1}, a \in$ $K$ is independent of the choice of $u$.

(2) The map $\theta: Q \rightarrow \operatorname{Aut}(K), x \mapsto \theta_{x}$ is a homomorphism.

Proof. (1).Let $u: Q \rightarrow G$, and $u^{\prime}: Q \rightarrow G, \pi u^{\prime}(x)=x, \pi u(x)=x$. Then $u(x) u^{\prime}(x) \in k e r \pi=K$ Therefore, $u^{\prime}(x)=u(x) b$ for some $b \in K$.Now $u^{\prime}(x) a u^{\prime}(x)^{-1}=u(x) a(u(x) b)^{-1}=u(x) b b^{-1} u(x)^{-1}=u(x) a u(x)^{-1}$ since $K$ is abelian.

(2). Since $K$ is normal in $G$, then $\theta_{x}(a)=u(x) a u(x)^{-1} \in K$. So $\theta_{x}$ is a map from $K$ to $K$. Also $\theta_{X}$ is an automorphism because conjugations are. If $x, y \in Q$,then

$$
\theta_{x}\left(\theta_{y}(a)\right)=\theta_{x}\left(u(x) a u(x)^{-1}\right)=u(x) u(y) a u(y)^{-1} u(x)^{-1}
$$

while

$$
\theta_{x y}(a)=u(x y) a u(x y)^{-1}
$$

But $u(x y)$ and $u(x) u(y)$ both are lifting of $x y, \pi u(x y)=\pi(u(x) u(y))$. So by part (1), $\theta_{x} \theta_{y}=\theta_{x y}$.

Remark. The homomorphism $\theta$ indicates how $K$ is normal in $G$. For example let $K$ be a cyclic group of order 3 and $Q=<x>$ be the cyclic group of order 2. If $G$ is the semidirect product, then $G$ is abelian and $K$ lies in the center of $G$. In this case $u(x) a u(x)^{-1}=a$ for all $a \in K$ and $\theta_{x}=1_{K}$.

proposition 1.5 Let $K$ and $Q$ be topological groups with $K$ abelian. Then $\theta: Q \rightarrow \operatorname{Aut}(K)$ makes $K$ into a $Z Q$-module $x a=\theta_{x}(a)$ for all $a \in Q$. Conversely if $K$ is a left $Z Q$-module then $x \mapsto \theta_{x}$ defines a homomorphism $\theta \rightarrow \operatorname{Aut}(K)$.

Proof.Let $b \in Z Q$.Then $b$ has a unique expression of the form $w=\sum_{x \in Q} m_{x} x$ where $m_{x} \in Z$ and almost all $m_{x}=0$. Define

$$
\left(\sum m_{x} x\right) a=\sum m_{x} \theta_{x}(a)=\sum m_{x}(x a)
$$


Since $\theta$ is a homomorphism , $\theta(1)=1+K$, and so $1 a=\theta_{1}(a)$. Since $\theta_{x} \in \operatorname{Aut}(K), x(a+b)=x a+x b$ It follows that $w(a+b)=w a+w b$ for all $w \in Z Q$. Similarly, $(w+v) a=w a+v a w, v \in Z Q$. Finally $(w v) a=w(v a)$ since $(x y) a=x(y a), x, y \in Q$. But $(x y) A=\theta_{x y}(a)=\theta_{x}\left(\theta_{y}(a)\right)=\theta_{x}(y a)=x(y a)$.

Corollary 1.6 If $0 \rightarrow K \stackrel{i}{\rightarrow} G \stackrel{\pi}{\rightarrow} Q \rightarrow 0$ is an extension with a continuous section $u: Q \rightarrow G$. Then $K$ is a left $Z Q$-module by $x a=u(x) a u(x)^{-1} x \in Q$, $a \in K$. The multiplication is independent of the choice of $u$.

Proof. By proposition 1.4 and 1.5.

Now we express The semidirect product as a product of groups.

Proposition 1.7 Let $K$ be a normal subgroup of $G$

(1) If $0 \rightarrow K \stackrel{i}{\rightarrow} G \stackrel{\pi}{\rightarrow} Q \rightarrow 0$ is a splitting with $j: Q \rightarrow G, \pi j=1_{Q}$, then $i(K) \cap j(Q)=0$ and $i(k) j(Q)=G$

(2) Every $g \in G$ has a unique form $g=a j(x), a \in K, x \in Q$

(3) If $K$ and $Q$ are subgroups of $G$ with $K$ normal in $G$ then $G$ is a semidirect product of $K$ by $Q$ iff $K \cap Q=\{1\}, K Q=G$ and each $g \in G$ has a unique form $g=a x, a \in K, x \in Q$

Proof. (1). If $g \in i(K) \cap j(Q)$, then $g=i(a)=j(x)$ for some $a \in$ $K, x \in Q$. Now $g=j(x)$ implies that $\pi(g)=\pi j(x)=x, \pi(g)=\pi i(a)=0$. Therefore, $x=0$ and $g=j(x)=0$. If $g \in G$ then $\pi(g)=\pi j \pi(g)$ and so $g j\left(\pi(g)^{-1}\right) \in k e r \pi$; hence there is $a \in K$ with $a(j \pi(g))^{-1}=i(a)$ and so $g=i(a) j(\pi(g)) \in(i(K)(j(Q))$.

(2) We identify $i(a)$ as $a$.If $g=a j(x)=a^{\prime} j\left(x^{\prime}\right)$, then $i(a) i\left(a^{\prime}\right)^{-1}=j(x) j\left(x^{\prime}\right)^{-1}$. Hence $0=\pi i(a) i\left(a^{\prime}\right)^{-1}=\pi j(x) j\left(x^{\prime}\right)^{-1}=x x^{\prime}$. So $x=x^{\prime}$. Similarly $a=a^{\prime}$.

(3) Since $i$ and $j$ are inclusions, necessity is the spacial case of (2). Conversely if each $g \in G$ has a unique expression $g=a x, a \in K, x \in Q$. Define $\pi: G \rightarrow Q$ by $\pi(a x)=x$ It is easy to check that $\pi$ is a continuous homomorphism.

Let $H$ and $N$ be topological groups and $\alpha: H \times N \rightarrow N$ be a continuous action of $H$ on $N$ i.e.

$$
\alpha: H \times N \rightarrow N, \alpha(h, n) \in \operatorname{Aut}(N), \forall h \in H
$$

and

$$
\alpha_{h h^{\prime}}=\alpha_{h} \cdot \alpha_{h^{\prime}} \forall h, h^{\prime} \in H
$$

In the following we show that there is a one to one correspondence between the actions and split extensions of topological groups. 
Proposition 1.8 If $H$ and $N$ are topological groups, then each action of $H$ on $N$ determines a split extension of $H$ by $N$.

Proof. Let $\alpha: H \rightarrow \operatorname{Aut}(N)$ be a continuous action of $H$ on $N$. We define the group $H \propto_{\alpha} N$ as follows: as a space $H \propto_{\alpha} N$ is the cartesian product $H \times G$ and multiplication is given by

$$
(h, n)\left(h^{\prime}, n^{\prime}\right)=\left(h h^{\prime}, \alpha_{H^{\prime}-1}(n) n^{\prime}\right)
$$

The inverse of $(h, n) \in H \propto_{\alpha} N$ is $\left(h^{-1}, \alpha_{h}(n)^{-1}\right)$.It is easy to show that $H \propto_{\alpha} G$ is a topological group. Let $q_{H}, q_{N}$ be the natural embedding of $H$ and $N$, respectively, and $P_{H},(h, n) \mapsto h, h \in H, n \in N$. Now

$$
\left(\varepsilon_{\alpha}\right): 0 \rightarrow N \rightarrow H \propto_{\alpha} N \stackrel{P_{H}}{\rightarrow} H \rightarrow 0
$$

is an extension with splitting homomorphism $q_{H}: H \rightarrow H \propto_{\alpha} N$.

Note. The group $H \propto_{\alpha} N$ is called the semidirect product of $H$ and $N$ with respect to $\alpha$.

Definition 1.9 let $H$ and $N$ be topological groups and $\alpha, \alpha^{\prime}$ be continuous actions of $H$ on $N$. The actions are equivalent,

$\alpha \sim \alpha^{\prime}$, if there is a continuous map $\gamma: H \rightarrow N$ such that

(1) $\alpha_{h}=\alpha_{h}^{\prime} \cdot S_{\gamma(h)}$

(2) $\gamma\left(h h^{\prime} \alpha_{h^{\prime}-1}(n)=\alpha_{h^{-1}}^{\prime}(\gamma(h) n)\left(\gamma\left(h^{\prime}\right)\right), h, h^{\prime} \in H, n \in N\right.$

where $S_{m}: N \rightarrow N$ is the automorphism $S_{m}(n)=m n m^{-1}, m \in H, n \in N$

Let $\operatorname{Act}(G, H)$ be the set of continuous actions of $G$ on $H$ and $\operatorname{Ext}_{\text {split }}(G, H)$ the set of equivalence of split extensions of $G$ by $H$.

Theorem 1.10 There is a one to one correspondence between $\operatorname{Act}(G, H)$ and $\operatorname{Ext}_{\text {split }}(G, H)$.

Proof. Let $\boldsymbol{\Delta}: \operatorname{Act}(G, H) \rightarrow \operatorname{Ext}_{\text {split }}(G, H)$ be defined by $\boldsymbol{\Delta}(\alpha)=\varepsilon_{\alpha}$ ,$\varepsilon_{\alpha}$ as in proposition 1.8 . Let $\alpha$ and $\alpha$ ' be two extensions of $G$ by $H$ and $\varepsilon_{\alpha}: H \rightarrow G \propto_{\alpha} H \rightarrow G, \varepsilon_{\alpha^{\prime}}: H \rightarrow G \propto_{\alpha^{\prime}} H \rightarrow G$ the split extensions induced by $\alpha$ and $\alpha^{\prime}$,respectively. If $\varepsilon_{\alpha} \sim \varepsilon_{\alpha^{\prime}}$, then there is a continuous isomorphism $s: G \propto_{\alpha} H \rightarrow G \propto_{\alpha^{\prime}} H$ such that the following diagram commutes:

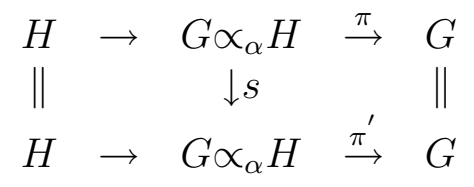


Then for $h \in G, n \in H$

$$
\begin{aligned}
\alpha_{h}(n) & =q_{H}^{-1}\left[q_{G}(h) q_{G}(h)^{-1}\right] \\
& =q_{H}^{\prime-1} s\left[s^{-1} q_{G}^{\prime}(h) \cdot s^{-1} q_{n}^{\prime}(n) \cdot s^{-1} q_{G}^{\prime} h^{-1}\right] \\
& =q_{H}^{\prime}\left[q_{h}^{\prime}(h) q_{n}^{\prime}(n) q_{h}^{\prime}(h)^{-1}\right] \\
& =\alpha_{h}^{\prime}(n)
\end{aligned}
$$

So the map $\Delta$ is one to one.

Now we show that the map is onto. Let $\varepsilon_{\alpha}: H \stackrel{f}{\rightarrow} Q \stackrel{q}{\rightarrow} G$ be the semidirect product of $G$ on $H$ with homomorphism $r: G \rightarrow Q, q r=I d_{H}$. Let $\alpha$ be the continuous action of $G$ on $H$, given by

$$
\alpha_{h}(n)=f^{-1}\left[r(h) f(n) r(h)^{-1}\right]
$$

This action induces the split extension $H \rightarrow G \propto H \rightarrow G$.It can easily be prove that $s: G \propto H \rightarrow Q, s(h, n)=r(h) f(n)$ is a continuous isomorphism which gives the equivalence of $\varepsilon$ and $\varepsilon_{\alpha}$. So $\Delta$ is onto.

As a result we have:

Theorem 1.11 Let $K$ be an abelian topological group. If $G$ is the semidirect product of $K$ by $Q$, then there is a $Q$-module structure on $K$ so that $G \simeq K \propto$ $Q$.

Proof. If $a \in K$ and $x \in Q$ we define $x . a=x a x^{-1}$. It is clear that the action is continuous and makes $K$ a $Q$-module. By theorem 1.10, this action determines a split extension. By proposition 1.7 each $g \in G$ can uniquely be expressed as $g=a x, a \in K, x \in Q$. Now we define $\phi: G \rightarrow K \propto Q$ by $a x \mapsto(a, x)$. A straight forward argument shows that $\phi$ is an isomorphism. Hence $G \simeq K \propto Q$.

\section{Vanishing of $H^{1}$}

Vanishing of $H^{2}(Q, K)$ implies that every extension of $Q$ by $K$ is a semidirect product. In this section we see that vanishing of $H^{1}(Q, K)$ implies conjugacy of complements when $K$ is abelian.

Definition 2.1. An automorphism $\phi: G \rightarrow G$ stabilizes an extension $0 \rightarrow$ $K \rightarrow G \rightarrow Q \rightarrow 0$ if the following diagram commutes:

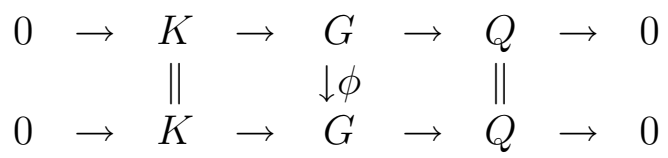


The set of all stabilizing of $K$ by $Q$, denoted by $\operatorname{Stab}(Q, K)$, is a group under composition.

Note that stabilizing automorphism is an isomorphism that gives an equivalence of an extension with itself. We will show that $\operatorname{Stab}(Q, K)$ does not depend on the extension.

Proposition 2.2 Let $K$ be abelian and $0 \rightarrow K \rightarrow G \stackrel{\pi}{\rightarrow} Q \rightarrow 0$ a split extension of topological groups with a continuous section $u: Q \rightarrow G$. Then every stabilizer $\phi: G \rightarrow G$ has the form

$$
\phi(a u(x))=a d(x) u(x)
$$

where $d(x) \in K$ is independent of the choice of $u$. Moreover (2.2.1) defines a stabilizing automorphism iff for all $x, y \in Q$ the map $d: Q \rightarrow K$ satisfies

$$
d(x y)=d(x)+x d(y)
$$

Proof. If $\phi$ is a stabilizing then $\pi \phi=\phi$. Since the extension splits so

$$
\phi(u(x))=d(x) u(y)
$$

for $d(x) \in K, y \in Q$. Then $x=\pi(u(x))=\pi \phi(u(x))=\pi(d(x) u(y))=y$. That is $x=y$. Therefore $\phi(a u(x))=\phi(a) \phi(u(x))=a d(x) u(x)$. The converse is easy.

Definition 2.3 . Let $Q$ be a topological group and $K$ a $Q$-module. A derivation (or crossed homomorphism) is a continuous map $d: Q \rightarrow K$ such that :

$$
d(x y)=d(x)+x d(y)
$$

The set of all derivations, denoted by $\operatorname{Der}(Q, K)$, is an abelian group under pointwise addition. If $K$ is a trivial $Q$-module then $\operatorname{Der}(Q, K)=\operatorname{Hom}_{Q}(Q, K)$. It is easy to see that $d(1)=1$.

A derivation of the form $d(x)=x a_{0}-a_{0}, a_{0} \in K$ is called a principal derivation. The set of principal derivation,$P \operatorname{Der}(Q, K)$, is a subgroup of $\operatorname{Der}(Q, K)$. By definition $H^{1}(Q, K)=\operatorname{Der}(Q, K) / P \operatorname{Der}(Q, K)$.

Proposition 2.4 Let $Q$ be a topological group , $K$ a $Q$-module and $0 \rightarrow$ $K \rightarrow G \rightarrow Q \rightarrow 0$ a split extension. There is a one to one correspondence between $\operatorname{Stab}(Q, K)$ and $\operatorname{Der}(Q, K)$.

Proof Let $\phi \in \operatorname{Stab}(Q, K)$. If $u: Q \rightarrow G$ is a section then by proposition 2.2 ,

$$
\phi(a u(x))=a d(x) u(x)
$$


where $d$ is a derivation. Since $d$ is independent of the choice of section so the map $\phi \mapsto d$ is well-defined, which easily seen to be a homomorpism.Now we construct an inverse map. If $d \in \operatorname{Der}(Q, K)$, define $\phi: G \rightarrow G$ by $\phi(a u(x))=\operatorname{ad}(x) u(x)$. By proposition $2.2, \phi$ is a stabilizer and $d \mapsto \phi$ is the desired inverse.

Remark. By proposition $2.4, H^{1}(Q, K)$ can be expressed as a quotient of inner automorphisms.

The next result characterizes the stabilizing of a semidirect product.

Let $K$ be an abelian group . We write the operation in $K$ additively.We use the following notational convention: Even though $G$ may not be abelian, additive notation will be used for the operation in $G$.

Proposition 2.5 Let $G$ be the semidirect product of $K$ by $Q$. Then $\phi$ : $G \rightarrow G$ is an inner stabilizing of the extension $0 \rightarrow K \rightarrow G \rightarrow Q \rightarrow 0$ with a continuous section $u: Q \rightarrow G$ iff

$$
\phi\left(a+u(x)=a+\left(x . a_{0}\right)-a_{0}+u(x)\right.
$$

for some $a_{0} \in K$.

Proof. Let $\phi(a+u(x))=a+x a_{0}-a_{0}+u(x)$ write $d(x)=x a_{0}-a_{0}$. The $\phi(a+u(x)=a+d(x)+u(x)$. But $d$ is principal derivation , so , by proposition $2.2, \phi$ is an stabilizing automorphism . Now $\phi$ is a conjugation by $a_{0}$ :

$$
-a_{0}+(a u(x))+a_{0}=-a_{0}+a+x a_{0}+u(x)=\phi(a+u(x))
$$

Conversely, let $\phi$ be a stabilizing conjugation. Now $\phi(a+u(x))=a+d(x)+u(x)$ and $\phi(a+u(x))=a_{0}+a+u(x)-a_{0}$, since $\phi$ is conjugation by $a_{0}$. But $a_{0}+a+u(x)-a_{0}=a_{0}+a-x a_{0}+u(x)$ so that $d(x)=a_{0}-x a_{0}$.

Remark. Associated with any extension $0 \rightarrow K \rightarrow G \rightarrow Q \rightarrow 0$ with a continuous section $u: Q \rightarrow G$, there is a continuous map $f: Q \times Q \rightarrow$ $K, f\left(x_{1}, x_{2}\right)=u\left(x_{1}\right) u\left(x_{2}\right) u\left(x_{1} x_{2}\right)^{-1}[6]$. This is called a factor set . When the extension splits, then there exists a $u$ which is a homomorphism ; the corresponding factor set is identically 0 . Hence factor sets describe how an extension differs from being a split extension. Also we have the cocycle identity [4]:

$$
f(x, y)+f(x y, z)=x f(y, z)+f(x, y z)
$$

In the following we show that vanishing of $H^{1}(Q, K)$ implies the conjugacy of complements ,in the semidirect product of topological groups, when $K$ is 
abelian.

Proposition 2.6 Let $G$ be the semidirect product of $K$ by $Q$ and $C, C^{\prime}$ the complements of $K$ in $G$. If $H^{1}(Q, K)=0$, then $C$ and $C^{\prime}$ are conjugate.

Proof.Let $0 \rightarrow K \rightarrow G \rightarrow Q \rightarrow 0$ be the split extension corresponding to semidirect product of $K$ and $Q$. with the continuous sections $u: Q \rightarrow G, u^{\prime}$ : $Q \rightarrow G$ such that $u(Q)=C, u^{\prime}(Q)=C^{\prime}$. Since $H^{1}(Q, K)=0$, the factor sets $f$ and $f^{\prime}$ determined by homomorphisms $u, u^{\prime}$ are identically zero. So $f-f^{\prime}=0$. But by the above remark, there is a continuous map $h: Q \rightarrow K$ ,$h(x)=u^{\prime}(x)-u(x)$ such that $0=f^{\prime}(x, y)-f(x, y)=x h(y)-h(x y)+h(x)$. Thus $h$ is a derivation. Since $H^{1}(Q, K)=0, h$ is a principal derivation: there is $a_{0} \in K$ with $u^{\prime}(x)-u(x)=h(x)=x a_{0}-a_{0}$ for all $x \in Q$. Since addition in $G$ satisfies $u^{\prime}(x)-a_{0}=-x a_{0}+u^{\prime}(x)$, we have $u(x)=a_{0}-x a_{0}+u^{\prime}(x)-a_{0}$. So $C=u(Q)$ and $C^{\prime}=u^{\prime}(Q)$ are conjugate via $a_{0}$.

\section{References}

[1] R. C. Alperin,H. Sahleh, Hopf,s formula and the Schur multiplicator for topological groups , Kyungpook .Math.Journal.31 (1) (1991), 35-71.

[2] O.Berndt, A caregorical definition of semidirect products ,Applied categorical structures. 6 (1998), 37-62.

[3] O.Berndt , On semidirect products of commutative Banach algebras,Quaest. Math. 17 (1994), 67-81.

[4] S.Eilenberg, S.MacLane, Cohomology theory in abstract groups I, Ann of math. 48 (1947), 51-78.

[5] A.R.Garzon ,H.Inassaridze , Semidirect products of categorical groups .Obstrection theory, HHA . 3(6) (2001), 111-138.

[6] S.T.Hu, Cohomology theory in topological groups,Michigan Math.J. 1(1) (1952), 11-59.

[7] T.W.Palmer , Banach Algebras and the General theory of *-algebras, Volum 1:Algebras and Banach Algebras, Cambridge ,Encyclopedia of mathematics and its applications 49, Cambridge University Press, 1978.

[8] A. Shtern , Remarks on Pseudochrscters and the Real Continuous Bounded Cohomology of Connected Locally Compact Groups, annals of Global analysis and Geometry. 20 (2001) , 199-221. 
[9] M.P.Thomas , principal ideals and semi-direct products in commutative Banach algebras, J.Funct.Anal. 101 (1991), 312-328.

Received: November 21, 2006 